\title{
A NOTE ON THE DIFFERENCE SCHEMES FOR HYPERBOLIC EQUATIONS
}

\author{
A. ASHYRALYEV AND P. E. SOBOLEVSKII
}

Received 26 March 2001

The initial value problem for hyperbolic equations $d^{2} u(t) / d t^{2}+A u(t)=f(t)$ $(0 \leq t \leq 1), u(0)=\varphi, u^{\prime}(0)=\psi$, in a Hilbert space $H$ is considered. The first and second order accuracy difference schemes generated by the integer power of $A$ approximately solving this initial value problem are presented. The stability estimates for the solution of these difference schemes are obtained.

\section{Introduction}

We consider the initial value problem

$$
\begin{gathered}
\frac{d^{2} u(t)}{d t^{2}}+A u(t)=f(t) \quad(0 \leq t \leq 1), \\
u(0)=\varphi, \quad u^{\prime}(0)=\psi,
\end{gathered}
$$

for a differential equation in a Hilbert space $H$ with unbounded linear selfadjoint and positive definite operator $A=A^{*} \geq \delta I(\delta>0)$ with dense domain $\bar{D}(A)=$ $H$. It is known (cf. [3]) that various initial boundary value problems for the hyperbolic equations can be reduced to problem (1.1). A study of discretization, over time only, of the initial value problem also permits one to include general difference schemes in applications, if the differential operator in space variables, $A$, is replaced by the difference operators $A_{h}$ that act in the Hilbert spaces and are uniformly positive definite and selfadjoint in $h$ for $0<h \leq h_{0}$. In the paper [4], the following first order accuracy difference scheme for approximately solving problem (1.1)

$$
\begin{gathered}
\tau^{-2}\left(u_{k+1}-2 u_{k}+u_{k-1}\right)+A u_{k+1}=f_{k}, \quad f_{k}=f\left(t_{k}\right), t_{k}=k \tau, \\
1 \leq k \leq N-1, \quad N \tau=1, \\
\tau^{-1}\left(u_{1}-u_{0}\right)+i A^{1 / 2} u_{1}=i A^{1 / 2} u_{0}+\psi, \quad u_{0}=\varphi,
\end{gathered}
$$

Copyright $(9) 2001$ Hindawi Publishing Corporation

Abstract and Applied Analysis 6:2 (2001) 63-70

2000 Mathematics Subject Classification: 35L, 34G, 65J, 65N

URL: http://aaa.hindawi.com/volume-6/S1085337501000501.html 
64 A note on the difference schemes for hyperbolic equations

was considered. The stability estimates for the solution of the difference scheme (1.2) were obtained. The proof of these statements is based on the transform of second order difference equations to equivalent system of first order difference equations. Application of this approach in $[1,2]$ with similar results for the solutions of the second order accuracy of the following difference schemes

$$
\begin{gathered}
\tau^{-2}\left(u_{k+1}-2 u_{k}+u_{k-1}\right)+A u_{k}+\frac{\tau^{2}}{4} A^{2} u_{k+1}=f_{k}, \\
f_{k}=f\left(t_{k}\right), \quad 1 \leq k \leq N-1, \quad N \tau=1, \\
\tau^{-1}\left(u_{1}-u_{0}\right)+i A^{1 / 2}\left(I+\frac{i \tau}{2} A^{1 / 2}\right) u_{1}=z_{1}, \\
z_{1}=\left(I+i \tau A^{1 / 2}\right) \psi+\frac{\tau}{2} f_{0}+\left(i A^{1 / 2}-\tau A\right) u_{0}, \quad f_{0}=f(0), u_{0}=\varphi, \\
\tau^{-2}\left(u_{k+1}-2 u_{k}+u_{k-1}\right)+\frac{1}{4} A\left(u_{k+1}+2 u_{k}+u_{k-1}\right)=f_{k}, \\
f_{k}=f\left(t_{k}\right), \quad 1 \leq k \leq N-1, \quad N \tau=1, \\
\tau^{-1}\left(u_{1}-u_{0}\right)+\frac{i}{2} A^{1 / 2}\left(u_{1}+u_{0}\right)=z_{1}, \\
z_{1}=\left(I+\frac{i \tau}{2} A^{1 / 2}\right) \psi+\frac{\tau}{2} f_{0}+\frac{1}{2}\left(i A^{1 / 2}-\tau A\right) u_{0}, \quad f_{0}=f(0), u_{0}=\varphi
\end{gathered}
$$

for approximately solving the initial value problem (1.1) were obtained. However, for practical realization of these difference schemes it is necessary to first construct an operator $A^{1 / 2}$. This action is very difficult for a realization. Therefore, in spite of theoretical results the role of their application to a numerical solution for an initial value problem is not great.

In the present paper, first and second order accuracy difference schemes for approximate solutions of problem (1.1) are constructed using the integer powers of the operator $A$, and the stability estimates for the solution of these difference schemes are obtained.

\section{First order difference schemes}

We consider the first order accuracy difference scheme for approximately solving the initial value problem (1.1)

$$
\begin{gathered}
\tau^{-2}\left(u_{k+1}-2 u_{k}+u_{k-1}\right)+A u_{k+1}=f_{k}, \quad f_{k}=f\left(t_{k}\right), t_{k}=k \tau, \\
1 \leq k \leq N-1, \quad N \tau=1, \\
\tau^{-1}\left(u_{1}-u_{0}\right)=\psi, \quad u_{0}=\varphi .
\end{gathered}
$$


THEOREM 2.1. Let $\varphi \in D(A), \psi \in D(A)$. Then for the solution of the difference scheme (2.1) the following stability inequalities, for $2 \leq k \leq N$, hold

$$
\begin{aligned}
&\left\|u_{k}\right\|_{H} \leq \tau \sum_{s=1}^{k-1}\left\|A^{-1 / 2} f_{S}\right\|_{H}+\left\|A^{-1 / 2} \psi\right\|_{H}+\|\varphi\|_{H}, \\
&\left\|A^{1 / 2} u_{k}\right\|_{H} \leq \tau \sum_{s=1}^{k-1}\left\|f_{S}\right\|_{H}+\left\|A^{1 / 2} \varphi\right\|_{H}+\|\psi\|_{H}, \\
&\left\|A u_{k}\right\|_{H} \leq 2 \sum_{s=2}^{k-1}\left\|f_{S}-f_{S-1}\right\|_{H}+\left\|f_{1}\right\|_{H}+\left\|A^{1 / 2} \psi\right\|_{H}+\|A \varphi\|_{H}, \\
&\left\|u_{1}\right\|_{H} \leq\|\varphi\|_{H}+\left\|\left(I+i \tau A^{1 / 2}\right) A^{-1 / 2} \psi\right\|_{H}, \\
&\left\|A^{1 / 2} u_{1}\right\|_{H} \leq\left\|A^{1 / 2} \varphi\right\|_{H}+\left\|\left(I+i \tau A^{1 / 2}\right) \psi\right\|_{H}, \\
&\left\|A u_{1}\right\|_{H} \leq\|A \varphi\|_{H}+\left\|\left(I+i \tau A^{1 / 2}\right) A^{1 / 2} \psi\right\|_{H} .
\end{aligned}
$$

The proof of this theorem uses the method of [4] and is based on the following formulas:

$$
\begin{aligned}
u_{1}= & \varphi+\tau \psi, \\
u_{k}= & \frac{1}{2}\left[R^{k-1}+\tilde{R}^{k-1}\right] \varphi+(R-\tilde{R})^{-1} \tau R\left(R^{k}-\tilde{R}^{k}\right) \psi-\sum_{s=1}^{k-1} \frac{\tau}{2 i} A^{-1 / 2}\left[R^{k-s}-\tilde{R}^{k-s}\right] f_{s} \\
= & \frac{1}{2}\left[R^{k-1}+\tilde{R}^{k-1}\right] \varphi+(R-\tilde{R})^{-1} \tau R\left(R^{k}-\tilde{R}^{k}\right) \psi \\
& +A^{-1} \frac{1}{2} \sum_{s=2}^{k-1}\left[R^{k-s}+\tilde{R}^{k-s}\right]\left(f_{s-1}-f_{s}\right)+2 f_{k-1}-\left[R^{k-1}+\tilde{R}^{k-1}\right] f_{1},
\end{aligned}
$$

for $2 \leq k \leq N$, where $R=\left(I+i \tau A^{1 / 2}\right)^{-1}, \tilde{R}=\left(I-i \tau A^{1 / 2}\right)^{-1}$ and on the estimates

$$
\begin{aligned}
\|R\|_{H \mapsto H} & \leq 1, & & \|\tilde{R}\|_{H \mapsto H} \leq 1, \\
\left\|R \tilde{R}^{-1}\right\|_{H \mapsto H} & \leq 1, & & \left\|\tilde{R} R^{-1}\right\|_{H \mapsto H} \leq 1, \\
\left\|\tau A^{1 / 2} R\right\|_{H \mapsto H} & \leq 1, & & \left\|\tau A^{1 / 2} \tilde{R}\right\|_{H \mapsto H} \leq 1 .
\end{aligned}
$$

Note that formulas (2.3) are generated by the operator $A^{1 / 2}$ and are used to prove stability estimates for the solutions of the difference scheme (2.1). 
66 A note on the difference schemes for hyperbolic equations

However, for the practical realization of this difference scheme (2.1) the operator $A^{1 / 2}$ as in [1, 2, 4] is not used. Note also that these stability inequalities in the case $k=1$ are weaker than the respective inequalities in the cases $k=2, \ldots, N$. However, obtaining this type of inequalities is important for applications. We denote by $a^{\tau}=\left(a_{k}\right)$ the mesh function of approximation. Then $\left\|\left(I+i \tau A^{1 / 2}\right) a_{1}\right\|_{H} \sim\left\|a_{1}\right\|_{H}=o(\tau)$ if we assume that $\tau\left\|A a_{1}\right\|_{H}$ tends to 0 as $\tau$ tends to 0 not slower than $\left\|a_{1}\right\|_{H}$. It takes place in applications by supplementary restriction on the smoothness property of the data in the space variables.

It is clear that the estimate

$$
\left\|u_{1}\right\|_{H} \leq\|\varphi\|_{H}+\left\|A^{-1 / 2} \psi\right\|_{H}
$$

is absent. However, estimates for the solution of first order accuracy modification difference scheme for approximately solving the initial value problem (1.1)

$$
\begin{gathered}
\tau^{-2}\left(u_{k+1}-2 u_{k}+u_{k-1}\right)+A u_{k+1}=f_{k}, \quad f_{k}=f\left(t_{k}\right), t_{k}=k \tau, \\
1 \leq k \leq N-1, \quad N \tau=1, \\
\left(I+\tau^{2} A\right) \tau^{-1}\left(u_{1}-u_{0}\right)=\psi, \quad u_{0}=\varphi,
\end{gathered}
$$

are better than the estimates for the solution of the difference scheme (2.1).

Theorem 2.2. Let $\varphi \in D(A), \psi \in D\left(A^{1 / 2}\right)$. Then for the solution of the difference scheme (2.6), the following stability inequalities, for $1 \leq k \leq N$, hold

$$
\begin{gathered}
\left\|u_{k}\right\|_{H} \leq \tau \sum_{s=1}^{k-1}\left\|A^{-1 / 2} f_{s}\right\|_{H}+\left\|A^{-1 / 2} \psi\right\|_{H}+\|\varphi\|_{H}, \\
\left\|A^{1 / 2} u_{k}\right\|_{H} \leq \tau \sum_{s=1}^{k-1}\left\|f_{s}\right\|_{H}+\left\|A^{1 / 2} \varphi\right\|_{H}+\|\psi\|_{H}, \\
\left\|A u_{k}\right\|_{H} \leq 2 \sum_{s=2}^{k-1}\left\|f_{S}-f_{s-1}\right\|_{H}+\left\|f_{1}\right\|_{H}+\left\|A^{1 / 2} \psi\right\|_{H}+\|A \varphi\|_{H} .
\end{gathered}
$$

The proof of this theorem is based on the following formulas:

$$
\begin{aligned}
u_{1}= & \varphi+\tau R \tilde{R} \psi, \\
u_{k}= & \frac{1}{2}\left[R^{k-1}+\tilde{R}^{k-1}\right] \varphi+(R-\tilde{R})^{-1} \tau R\left(R^{k}-\tilde{R}^{k}\right) R \tilde{R} \psi \\
& -\sum_{s=1}^{k-1} \frac{\tau}{2 i} A^{-1 / 2}\left[R^{k-s}-\tilde{R}^{k-s}\right] f_{s}
\end{aligned}
$$




$$
\begin{aligned}
= & \frac{1}{2}\left[R^{k-1}+\tilde{R}^{k-1}\right] \varphi+(R-\tilde{R})^{-1} \tau R\left(R^{k}-\tilde{R}^{k}\right) R \tilde{R} \psi \\
& +A^{-1} \frac{1}{2} \sum_{s=2}^{k-1}\left[R^{k-s}+\tilde{R}^{k-s}\right]\left(f_{s-1}-f_{s}\right) \\
& +2 f_{k-1}-\left[R^{k-1}+\tilde{R}^{k-1}\right] f_{1}, \quad 2 \leq k \leq N
\end{aligned}
$$

and on the estimates (2.4).

\section{Second order difference schemes}

We consider the second order accuracy difference schemes for approximate solutions of the initial value problem (1.1)

$$
\begin{gathered}
\tau^{-2}\left(u_{k+1}-2 u_{k}+u_{k-1}\right)+A u_{k}+\frac{\tau^{2}}{4} A^{2} u_{k+1}=f_{k}, \\
f_{k}=f\left(t_{k}\right), \quad t_{k}=k \tau, 1 \leq k \leq N-1, \quad N \tau=1, \\
\left(I+\tau^{2} A\right) \tau^{-1}\left(u_{1}-u_{0}\right)=\frac{\tau}{2}\left(f_{0}-A u_{0}\right)+\psi, \quad f_{0}=f(0), u_{0}=\varphi, \\
\tau^{-2}\left(u_{k+1}-2 u_{k}+u_{k-1}\right)+\frac{1}{2} A u_{k}+\frac{1}{4} A\left(u_{k+1}+u_{k-1}\right)=f_{k}, \\
f_{k}=f\left(t_{k}\right), \quad t_{k}=k \tau, 1 \leq k \leq N-1, \quad N \tau=1, \\
\left(I+\tau^{2} A\right) \tau^{-1}\left(u_{1}-u_{0}\right)=\frac{\tau}{2}\left(f_{0}-A u_{0}\right)+\psi, \quad f_{0}=f(0), u_{0}=\varphi .
\end{gathered}
$$

The stability estimates for the solution of these difference schemes are obtained.

THEOREM 3.1. Let $\varphi \in D(A), \psi \in D\left(A^{1 / 2}\right)$. Then for the solution of the difference scheme (3.1), the following stability inequalities, for $1 \leq k \leq N$, hold

$$
\begin{gathered}
\left\|u_{k}\right\|_{H} \leq \tau \sum_{s=0}^{k-1}\left\|A^{-1 / 2} f_{s}\right\|_{H}+\left\|A^{-1 / 2} \psi\right\|_{H}+\|\varphi\|_{H}, \\
\left\|A^{1 / 2} u_{k}\right\|_{H} \leq \tau \sum_{s=0}^{k-1}\left\|f_{s}\right\|_{H}+\left\|A^{1 / 2} \varphi\right\|_{H}+\|\psi\|_{H}, \\
\left\|A u_{k}\right\|_{H} \leq 2 \sum_{s=1}^{k-1}\left\|f_{s}-f_{s-1}\right\|_{H}+\left\|f_{0}\right\|_{H}+\left\|A^{1 / 2} \psi\right\|_{H}+\|A \varphi\|_{H} .
\end{gathered}
$$


68 A note on the difference schemes for hyperbolic equations

The proof of this theorem is based on the following formulas:

$$
\begin{aligned}
& u_{1}=\left(I+\tau^{2} A^{-1}\right)\left[\left(I+\frac{\tau^{2}}{2} A\right) \varphi+\tau \psi+\frac{\tau^{2}}{2} f_{0}\right] \\
& u_{k}=\left[R^{k}+\tau R(R-\tilde{R})^{-1}\left[R^{k}-\tilde{R}^{k}\right]\left(I+\tau^{2} A\right)^{-1}\left(-\tau A+i A^{1 / 2}\right)\right] \varphi \\
& +\tau R(R-\tilde{R})^{-1}\left[R^{k}-\tilde{R}^{k}\right]\left(I+\tau^{2} A\right)^{-1} \psi \\
& +\frac{\tau^{2}}{2} R(R-\tilde{R})^{-1}\left[R^{k}-\tilde{R}^{k}\right]\left(I+\tau^{2} A\right)^{-1} f_{0}-\sum_{s=1}^{k-1} \frac{\tau}{2 i} A^{-1 / 2}\left[R^{k-s}-\tilde{R}^{k-s}\right] f_{s} \\
& =\left[R^{k}+\tau R(R-\tilde{R})^{-1}\left[R^{k}-\tilde{R}^{k}\right]\left(I+\tau^{2} A\right)^{-1}\left(-\tau A+i A^{1 / 2}\right)\right] \varphi \\
& +\tau R(R-\tilde{R})^{-1}\left[R^{k}-\tilde{R}^{k}\right]\left(I+\tau^{2} A\right)^{-1} \psi \\
& +\frac{\tau^{2}}{2} R(R-\tilde{R})^{-1}\left[R^{k}-\tilde{R}^{k}\right]\left(I+\tau^{2} A\right)^{-1} f_{0} \\
& +A^{-1} \frac{1}{2} \sum_{s=1}^{k-1}\left[\left(I+\frac{i \tau}{2} A^{1 / 2}\right)^{-1} R^{k-s}+\left(I-\frac{i \tau}{2} A^{1 / 2}\right)^{-1} \tilde{R}^{k-s}\right]\left(f_{s-1}-f_{s}\right) \\
& +2\left(I+\frac{i \tau}{2} A^{1 / 2}\right)^{-1}\left(I-\frac{i \tau}{2} A^{1 / 2}\right)^{-1} f_{k-1} \\
& -\left[\left(I+\frac{i \tau}{2} A^{1 / 2}\right)^{-1} R^{k-1}+\left(I-\frac{i \tau}{2} A^{1 / 2}\right)^{-1} \tilde{R}^{k-1}\right] f_{0}, \quad 2 \leq k \leq N,
\end{aligned}
$$

where $R=\left(I+i \tau A^{1 / 2}-\left(\tau^{2} / 2\right) A\right)^{-1}, \tilde{R}=\left(I-i \tau A^{1 / 2}-\left(\tau^{2} / 2\right) A\right)^{-1}$ and on the estimates

$$
\begin{gathered}
\|R\|_{H \mapsto H} \leq 1, \quad\|\tilde{R}\|_{H \mapsto H} \leq 1, \quad\left\|R \tilde{R}^{-1}\right\|_{H \mapsto H} \leq 1, \\
\left\|\tilde{R} R^{-1}\right\|_{H \mapsto H} \leq 1, \quad\left\|\left(I \pm \frac{i \tau}{2} A^{1 / 2}\right)^{-1}\right\|_{H \mapsto H} \leq 1, \\
\left\|\left(I \pm i \tau A^{1 / 2}\right)^{-1}\right\|_{H \mapsto H} \leq 1, \quad\left\|\tau A^{1 / 2}\left(I \pm i \tau A^{1 / 2}\right)^{-1}\right\|_{H \mapsto H} \leq 1 .
\end{gathered}
$$


THEOREM 3.2. Let $\varphi \in D(A), \psi \in D\left(A^{1 / 2}\right)$. Then for the solution of the difference scheme (3.2), the following stability inequalities, for $1 \leq k \leq N$, hold

$$
\begin{gathered}
\left\|u_{k}\right\|_{H} \leq \tau \sum_{s=0}^{k-1}\left\|A^{-1 / 2} f_{S}\right\|_{H}+\left\|A^{-1 / 2} \psi\right\|_{H}+\|\varphi\|_{H}, \\
\left\|A^{1 / 2} u_{k}\right\|_{H} \leq \tau \sum_{s=0}^{k-1}\left\|f_{S}\right\|_{H}+\left\|A^{1 / 2} \varphi\right\|_{H}+\|\psi\|_{H}, \\
\left\|\frac{A\left(u_{k}+u_{k-1}\right)}{2}\right\|_{H} \leq 2 \sum_{s=1}^{k-1}\left\|f_{S}-f_{s-1}\right\|_{H}+\left\|f_{0}\right\|_{H}+\left\|A^{1 / 2} \psi\right\|_{H}+\|A \varphi\|_{H} .
\end{gathered}
$$

The proof of this theorem is based on the following formulas:

$$
\begin{aligned}
u_{1}= & \left(I+\tau^{2} A\right)^{-1}\left[\left(I+\frac{\tau^{2}}{2} A\right) \varphi+\tau \psi+\frac{\tau^{2}}{2} f_{0}\right], \\
u_{k}= & {\left[R^{k}+\frac{1}{2 i} A^{-1 / 2}\left(I-\frac{i \tau A^{1 / 2}}{2}\right)\left[R^{k}-\tilde{R}^{k}\right]\right.} \\
& \left.\times\left(\left(I+\frac{i \tau A^{1 / 2}}{2}\right) \frac{\tau}{2} A-i \tau A^{1 / 2}\left(I+\tau^{2} A\right)\right)\left(I+\tau^{2} A\right)^{-1}\right] \varphi \\
& +\frac{i}{2} A^{-1 / 2}\left(I-\frac{i \tau A^{1 / 2}}{2}\right)\left[R^{k}-\tilde{R}^{k}\right]\left(I+\tau^{2} A\right)^{-1}\left(I+\frac{i \tau A^{1 / 2}}{2}\right) \psi \\
& +\frac{i}{2} A^{-1 / 2}\left(I-\frac{i \tau A^{1 / 2}}{2}\right)\left[R^{k}-\tilde{R}^{k}\right]\left(I+\tau^{2} A\right)^{-1}\left(I+\frac{i \tau A^{1 / 2}}{2}\right) \frac{\tau}{2} f_{0} \\
& -\sum_{s=1}^{k-1} \frac{\tau}{2 i} A^{-1 / 2}\left[R^{k-s}-\tilde{R}^{k-s}\right] f_{s} \\
= & {\left[R^{k}+\frac{1}{2 i} A^{-1 / 2}\left(I-\frac{i \tau A^{1 / 2}}{2}\right)\left[R^{k}-\tilde{R}^{k}\right]\right.} \\
& \left.\times\left(\left(I+\frac{i \tau A^{1 / 2}}{2}\right) \frac{\tau}{2} A-i \tau A^{1 / 2}\left(I+\tau^{2} A\right)\right)\left(I+\tau^{2} A\right)^{-1}\right] \varphi \\
+ & \frac{i}{2} A^{-1 / 2}\left(I-\frac{i \tau A^{1 / 2}}{2}\right)\left[R^{k}-\tilde{R}^{k}\right]\left(I+\tau^{2} A\right)^{-1}\left(I+\frac{i \tau A^{1 / 2}}{2}\right) \psi \\
& \frac{i}{2} A^{-1 / 2}\left(I-\frac{i \tau A^{1 / 2}}{2}\right)\left[R^{k}-\tilde{R}^{k}\right]\left(I+\tau^{2} A\right)^{-1}\left(I+\frac{i \tau A^{1 / 2}}{2}\right) \frac{\tau}{2} f_{0}
\end{aligned}
$$


70 A note on the difference schemes for hyperbolic equations

$$
\begin{aligned}
& +A^{-1} \frac{1}{2} \sum_{s=1}^{k-1}\left[\left(I-\frac{i \tau A^{1 / 2}}{2}\right) R^{k-s}+\left(I+\frac{i \tau A^{1 / 2}}{2}\right) \tilde{R}^{k-s}\right]\left(f_{s-1}-f_{s}\right) \\
& +2 f_{k-1}-\left[\left(I-\frac{i \tau A^{1 / 2}}{2}\right) R^{k-1}+\left(I+\frac{i \tau A^{1 / 2}}{2}\right) \tilde{R}^{k-1}\right] f_{0}, \quad 2 \leq k \leq N,
\end{aligned}
$$

where $R=\left(I-i \tau A^{1 / 2} / 2\right)\left(I+i \tau A^{1 / 2} / 2\right)^{-1}, \tilde{R}=\left(I+i \tau A^{1 / 2} / 2\right)\left(I-i \tau A^{1 / 2} / 2\right)^{-1}$ and on the estimates

$$
\begin{gathered}
\|R\|_{H \mapsto H} \leq 1, \quad\|\tilde{R}\|_{H \mapsto H} \leq 1, \quad\left\|\left(I \pm \frac{i \tau A^{1 / 2}}{2}\right)^{-1}\right\|_{H \mapsto H} \leq 1, \\
\left\|\left(I \pm i \tau A^{1 / 2}\right)^{-1}\right\|_{H \mapsto H} \leq 1, \quad\left\|\tau A^{1 / 2}\left(I \pm i \tau A^{1 / 2}\right)^{-1}\right\|_{H \mapsto H} \leq 1 .
\end{gathered}
$$

\section{References}

[1] A. Ashyralyev and I. Muradov, On one difference scheme of a second order of accuracy for hyperbolic equations, Trudy Instituta Matematiki i Mechaniki Akad. Nauk Turkmenistana (Ashgabat), no. 1, 1995, pp. 58-63 (Russian).

[2] _ On difference schemes of a second order of accuracy for hyperbolic equations, Modelling Processes of Explotation of Gas Places and Applied Problems of Theoretical Gasohydrodynamics, Ashgabat, Ilym, 1998, pp. 127-138 (Russian).

[3] S. G. Kreı̆n, Lineikhye Differentsialnye Uravneniya v Banakhovom Prostranstve [Linear Differential Equations in a Banach Space], Izdat. "Nauka", Moscow, 1967 (Russian). MR 40\#508.

[4] P. E. Sobolevskii and L. M. Chebotaryeva, Approximate solution by method of lines of the Cauchy problem for abstract hyperbolic equations, Izv. Vyssh. Uchebn. Zaved. Mat. (1977), no. 5, 103-116 (Russian).

A. Ashyralyev: Department of Mathematics, Fatih University, Istanbul, TURKEY

Current address: International Turkmen-Turkish University, Ashgabat, TURKMENISTAN

E-mail address: aashyr@fatih.edu.tr

P. E. SobolevskiI: Institute of Mathematics, Hebrew University, Jerusalem, ISRAEL

E-mail address: pavels@math.hiji.ac.il 


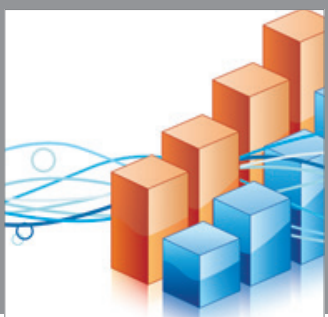

Advances in

Operations Research

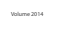

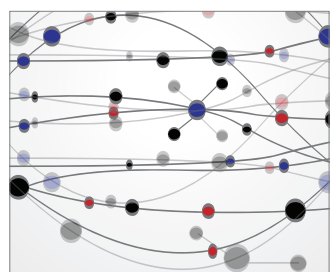

\section{The Scientific} World Journal
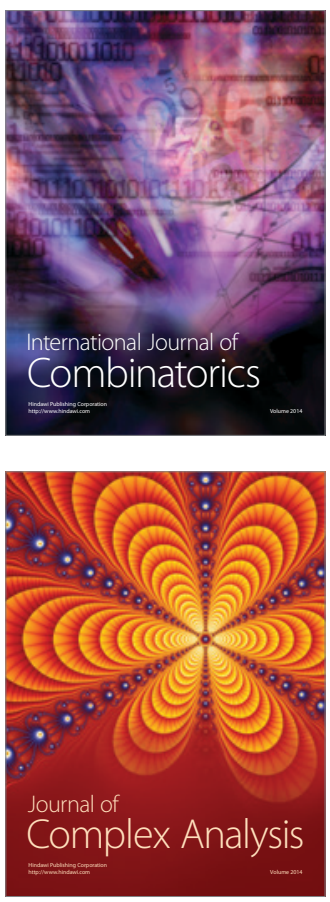

International Journal of

Mathematics and

Mathematical

Sciences
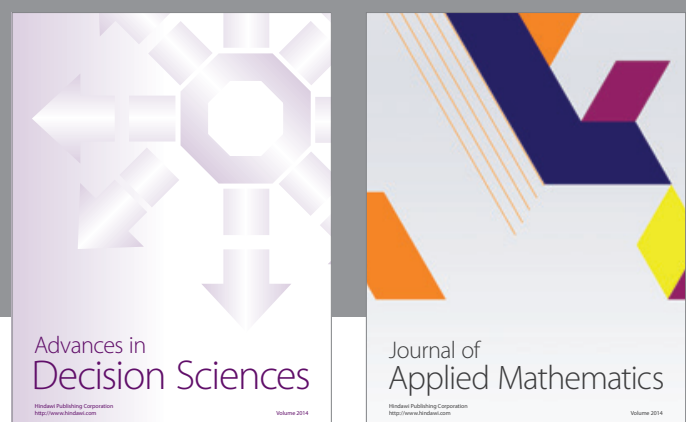

Journal of

Applied Mathematics
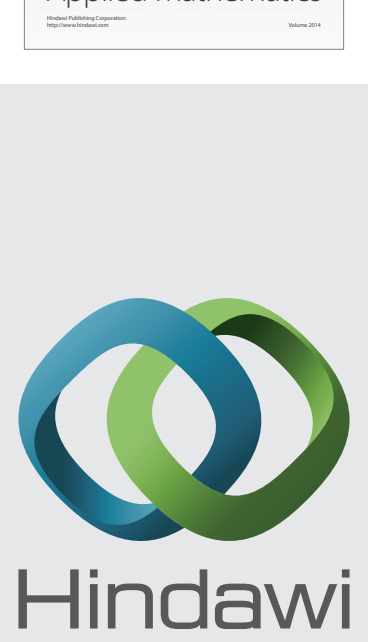

Submit your manuscripts at http://www.hindawi.com
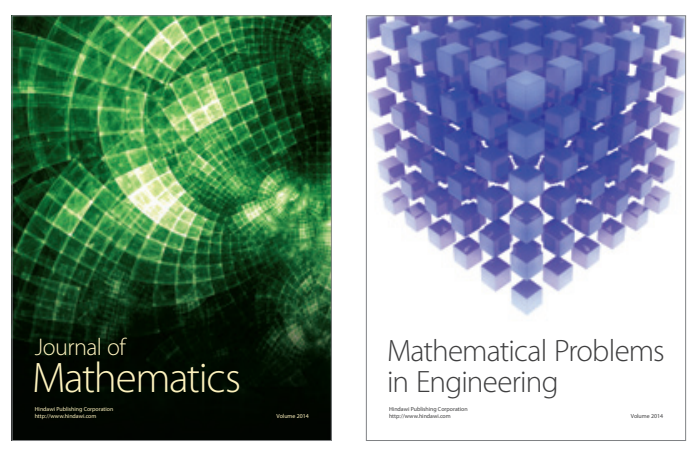

Mathematical Problems in Engineering
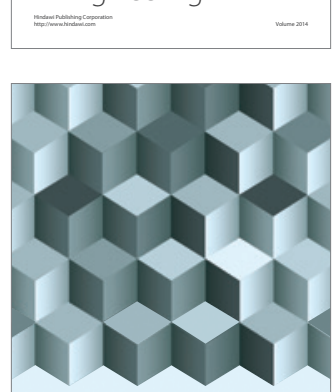

Journal of

Function Spaces
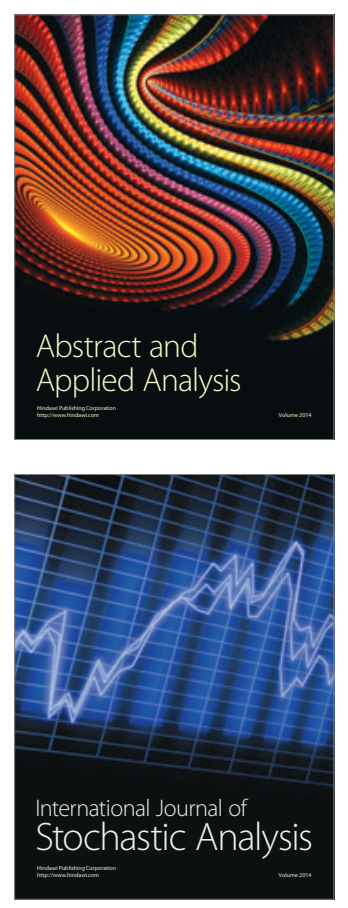

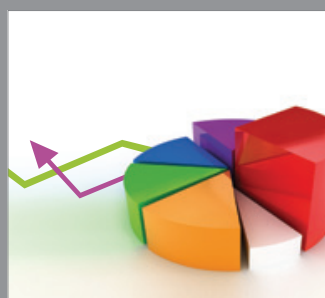

ournal of

Probability and Statistics

Promensencen
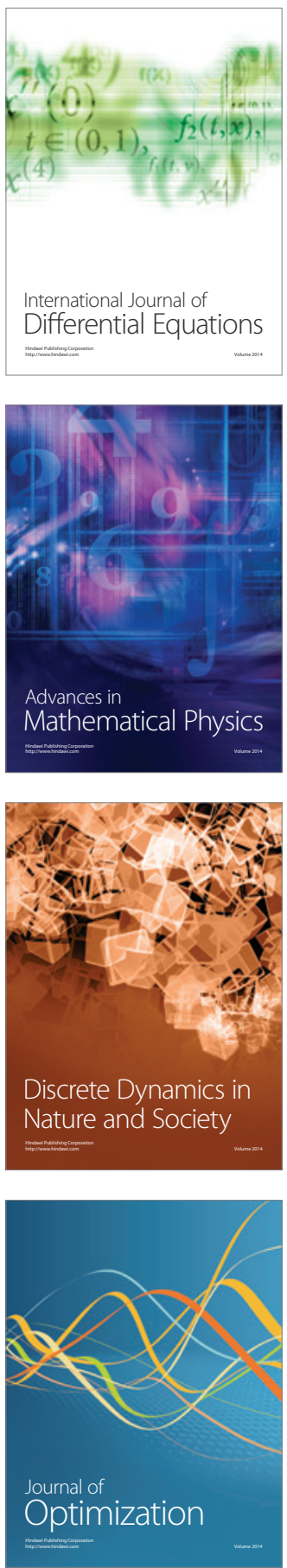